\title{
Metastatic potential in MDA-MB-231 human breast cancer cells is inhibited by proton beam irradiation via the Akt/nuclear factor- $\kappa B$ signaling pathway
}

\author{
KYU-SHIK LEE, DO-HYUNG LEE, SO-YOUNG CHUN and KYUNG-SOO NAM \\ Department of Pharmacology, College of Medicine, Dongguk University, Gyeongju 780-714, Republic of Korea
}

Received January 14, 2014; Accepted May 9, 2014

DOI: $10.3892 / \mathrm{mmr} .2014 .2259$

\begin{abstract}
A previous study has revealed that proton beam irradiation affects cell migration in MDA-MB-231 human breast cancer cells. Cyclooxygenase-2 (COX-2) and matrix metalloproteinase-9 (MMP-9) are highly expressed in various cancers, such as colon, lung and breast cancer, and enhance cell migration and metastasis in vitro and in vivo. In the present study, the effects of proton beam irradiation on COX-2 and MMP-9 expression levels in MDA-MB-231 human breast cancer cells were investigated, along with the signaling pathway involved in the proton beam irradiation-mediated antimetastatic effect. The results revealed that 12-O-tetradecanoylphorbol-13-acetate-induced increases in COX-2 and MMP-9 expression levels were reversed by proton beam irradiation in a dose-dependent manner. In addition, proton beam irradiation inhibited phosphorylation of protein kinase $\mathrm{B}$ (also known as Akt) and nuclear factor- $\mathrm{B}$ $(\mathrm{NF}-\kappa \mathrm{B})$, which are activated by phosphoinositide 3-kinase (PI3K) stimulation. MMP-9 and COX-2 expression levels are regulated by $\mathrm{PI} 3 \mathrm{~K} / \mathrm{Akt}$ and/or protein kinase $\mathrm{C} / \mathrm{mitogen}$-activated protein kinase signaling pathways that enhance $\mathrm{NF}-\kappa \mathrm{B}$ and activator protein-1 transcriptional activities. Therefore, the results suggest that proton beam irradiation inhibited the cancer cell growth and metastasis associated with COX-2 and MMP-9 expression in MDA-MB-231 human breast cancer cells, and that the antimetastatic effect of proton beam irradiation is achieved by the suppression of NF- $\kappa \mathrm{B}$ phosphorylation via inhibition of Akt activation.
\end{abstract}

\section{Introduction}

Due to low scattering properties in exposed tissue and deposition of the ionizing energy at an exact depth, proton beam irradiation is a useful tool in tumor radiotherapy, with no

Correspondence to: Professor Kyung-Soo Nam, Department of Pharmacology, College of Medicine, Dongguk University, 123 Dongdae-ro, Gyeongju 780-714, Republic of Korea

E-mail:namks@dongguk.ac.kr

Key words: proton beam, breast cancer, metastasis, cyclooxygenase-2, matrix metalloproteinase-9 radiation penetrating the normal tissue neighboring the tumor. Once a proton beam enters the body, the increased Bragg peak, the specific energy per unit at the end of the beam's range, induces excellent localization to the target $(1,2)$.

The mortality rates and prognoses in cancer patients are determined by the metastatic potential of the tumor. The five-year survival rate in localized breast cancer patients is $298 \%$; by contrast, only $27 \%$ of patients diagnosed with metastatic breast cancer survive for five years or longer (3). Therefore, prevention of metastasis is a required strategy to enhance the five-year survival rate for patients.

Metastasis is a multistep series of events that involves cancer cell dissociation from the primary tumor and invasion and seeding at a distant site $(4,5)$. The metastatic potential of tumor cells is closely associated with the expression levels of numerous proteins, including matrix metalloproteinases (MMPs), plasminogen activator (PA), nitric oxide synthase and cyclooxygenase (COX). COX catalyzes the synthesis of prostaglandins from arachidonic acid and exists in two predominant isoforms: COX-1, a constitutive enzyme, and COX-2, an inducible protein. COX-2 accelerates cancer progression and metastasis, and is overexpressed in various cancer types, including breast, colon, lung and gastric cancer $(6,7)$. Several studies have reported that cancer cell proliferation and metastasis are enhanced in the COX-2-overexpression system and are reduced by downregulation of COX-2 expression by inhibitors (8-11). Other studies have shown that MMP and vesicular endothelial growth factor expression levels are regulated by COX-2 $(10,12)$. The results of these studies indicate that COX-2 inhibition is important in the prevention of cancer development, proliferation and metastasis.

The invasiveness of breast cancer cells is reduced by COX-2 and MMP inhibition via the prevention of mitogen activated protein kinase (MAPK) or phosphoinositide 3-kinase (PI3K)/Akt signaling (10). By contrast, the metastatic potential of breast cancer cells is increased via the upregulation of COX-2 and MMPs by 12-O-tetradecanoylphorbol-13-acetate (TPA), activating the protein kinase $\mathrm{C}$ (PKC)/MAPK and PI3K/Akt signaling pathways (13-15). The enhancement of COX-2 and MMP-9 expression levels by TPA requires nuclear factor $-\kappa \mathrm{B}(\mathrm{NF}-\kappa \mathrm{B})$ and activator protein-1 (AP-1), which bind to the COX-2 and MMP-9 promoters (16-18). Numerous genes involved in cell proliferation, apoptosis, metastasis, cancer development and inflammation are governed by 
$\mathrm{NF}-\kappa \mathrm{B}$ and AP-1, which are activated by internal and external stimuli (19-21). The metastatic potential of numerous types of cancer cells has been shown to be determined by MMP-9 and COX-2 activities underlying the change in NF- $\kappa \mathrm{B}$ and/or AP-1 transcriptional activity $(15,20-22)$. The transcriptional activities of $\mathrm{NF}-\kappa \mathrm{B}$ and $\mathrm{AP}-1$ regulating COX-2 and MMP-9 are closely associated with the PKC/MAPK and PI3K/Akt signaling pathways (19-23).

Previous studies have revealed that the metastatic potential in MDA-MB-231 and MCF-7 human breast cancer cells was reduced by proton beam irradiation (14). In the present study, the molecular biological mechanism of the antimetastatic activity of proton beam irradiation in MDA-MB-231 human breast cancer cells was investigated.

\section{Materials and methods}

Cell culture. MDA-MB-231 human breast cancer cells were purchased from the Korean Cell Line Bank (Seoul, Korea). The cells were grown as a monolayer in Dulbecco's modified Eagle's medium (DMEM) supplemented with $10 \%$ fetal bovine serum and $1 \%$ antibiotic-antimycotic solution at $5 \% \mathrm{CO}_{2}$ and $37^{\circ} \mathrm{C}$. The cells were serum-starved for $24 \mathrm{~h}$ in serum-free DMEM medium prior to proton beam irradiation.

Proton beam irradiation. Proton beam irradiation was performed with 35-MeV proton beams at the MC-50-cyclotron of the Korean Institute of Radiological Sciences (Seoul, Korea) (14). The cells were irradiated at the center of the Bragg peaks, modulated to $6 \mathrm{~cm}$ widths.

Semi-quantitative reverse transcription polymerase chain reaction $(R T-P C R)$. A total of $5 \times 10^{5}$ cells/well of MDA-MB-231 human breast cancer cells were seeded on 6-well plates and grown for $24 \mathrm{~h}$ at $37^{\circ} \mathrm{C}$ in a $5 \%(\mathrm{v} / \mathrm{v}) \mathrm{CO}_{2}$ atmosphere. Following serum-starvation for $24 \mathrm{~h}$, the cells were irradiated with a proton beam and then cultured for an additional $24 \mathrm{~h}$, with or without $100 \mathrm{nM}$ TPA. The cells were collected by centrifugation following trypsinization. Total RNA was extracted by using the easy-BLUE ${ }^{\mathrm{TM}}$ Total RNA Extraction kit (iNtRON Biotechnology, Sungnam, Korea) according to the manufacturer's instructions. RT-PCR was conducted with the Avian Myeloblastosis Virus RNA PCR kit version 3.0 (Takara Bio, Inc., Shiga, Japan) and $1 \mu \mathrm{g}$ total RNA. The primer sequences were as follows: MMP-9: Forward 5'-TTCATCTTCCAAGGC CAATC-3' and reverse 5'-CTTGTCGCTGTCAAAGTTCG-3' (annealing temperature, $55^{\circ} \mathrm{C}$ ) (24); COX-2: Forward 5'-TTC ACGCATCAGTTTTTCAA-3' and reverse 5'-ACAGCAAAC CGTAGATGCTC-3' (annealing temperature, $55^{\circ} \mathrm{C}$ ) (25); GAPDH: Forward 5'-ATCCCATCACCATCTTCCAG-3' and reverse 5'-TTCTAGACGGCAGGTCAGGT-3' (annealing temperature, $\left.58^{\circ} \mathrm{C}\right)(26)$. The PCR products were subjected to $1.2 \%$ agarose gel electrophoresis containing $0.5 \mu \mathrm{g} / \mathrm{ml}$ ethidium bromide and were visualized on a UV transluminometer (CoreBio System, Seoul, Korea). Bands were densitometrically analyzed using Scion Image (Scion Corporation, Frederick, MD, USA).

Nuclear fractionation. The cells were washed twice with phosphate-buffered saline (PBS), and hypotonic buffer [containing
$20 \mathrm{mM}$ Tris- $\mathrm{HCl}$ (pH 7.4), $10 \mathrm{mM} \mathrm{NaCl}$ and $3 \mathrm{mM} \mathrm{MgCl}_{2}$, plus protease inhibitor cocktail and phosphatase inhibitor cocktail] was added to each sample. The cells were scraped with a rubber policeman and held on ice for $15 \mathrm{~min}$; then 1/8 volume $10 \%$ NP-40 was added. The cells were vortexed for $10 \mathrm{sec}$ at the maximum setting and centrifuged for $10 \mathrm{~min}$ at $2,500 \mathrm{x} \mathrm{g}$ at $4^{\circ} \mathrm{C}$ after $10 \mathrm{~min}$ incubation on ice. The supernatants were removed and designated as the cytosol fraction and the pellets were designated as the nuclear fraction. The pellets were washed with hypotonic buffer and then lysed with Cell Extraction Buffer (Invitrogen Life Technologies, Carlsbad, CA, USA), containing protease inhibitor cocktail and phosphatase inhibitor cocktail (GenDepot, Barker, TX, USA), for $30 \mathrm{~min}$ on ice, vortexing at 10 -min intervals. The lysates were separated by centrifugation at $14,000 \times \mathrm{g}$ for $30 \mathrm{~min}$ at $4^{\circ} \mathrm{C}$; the supernatants were subsequently removed, stored at $-80^{\circ} \mathrm{C}$, and labeled as the nuclear fractions.

Preparation of total cell lysate and western blotting. The cells were lysed in radioimmunoprecipitation assay lysis buffer containing phosphatase and protease inhibitor cocktails. Total cell lysates were prepared by centrifugation of the lysed cells at $14,000 \times \mathrm{g}$ for $10 \mathrm{~min}$ at $4{ }^{\circ} \mathrm{C}$ and stored at $-80^{\circ} \mathrm{C}$. The protein concentrations in the total cell lysates were determined by the bicinchoninic acid method. The protein samples were subjected to SDS-PAGE and transferred onto polyvinylidene fluoride membranes. The membranes were blocked with 5\% skimmed milk in Tris-buffered saline containing $0.1 \%$ Tween-20 and then incubated with primary antibody $(1: 3,000)$ overnight at $4^{\circ} \mathrm{C}$; anti-mouse monoclonal COX-2 (1:3,000; Invitrogen Life Technologies) and $\beta$-actin (1:3,000; Santa Cruz Biotechnology, Dallas, TX, USA). Antirabbit monoclonal Akt, p-Akt, Erk1/2, p-Erk1/2, JNK1/2, p-JNK1/2, p38, p-p38, NF- $\kappa$ B, p-NF- $\mathrm{B}$, c-Jun and lamin B (1:3,000; Cell Signaling Technology, Beverly, MA, USA). Subsequently, the membranes were incubated for $1 \mathrm{~h}$ at room temperature with secondary antibodies; goat anti-mouse and -rabbit IgG HRP (1:3,000; Santa Cruz Biotechnology). Protein bands were visualized with West-Q Chemiluminescent Substrate Kit Plus (GenDepot) and images were captured by the Luminescent Image Analyzer LAS-4000 (Fujifilm, Tokyo, Japan). The band densities were densitometrically analyzed by Scion Image.

\section{Results}

Changes in COX-2 and MMP-9 expression levels following proton beam irradiation. In a previous study, cell migration and MMP-9 activity in MDA-MB-231 human breast cancer cells induced by TPA were shown to be dose-dependently reduced by proton bream irradiation (14). MMP-9 activity is determined by its expression levels and is important in metastasis. Furthermore, MMP-9 expression levels are closely associated with COX-2 expression levels. Therefore, COX-2 expression levels may be regulated and MMP-9 transcription reduced by proton beam irradiation. In the present study, the effects of proton beam irradiation on COX-2 expression levels and MMP-9 transcription were investigated in MDA-MB-231 human breast cancer cells. As shown in Fig. 1A and B, proton beam irradiation inhibited TPA-induced COX-2 and MMP-9 

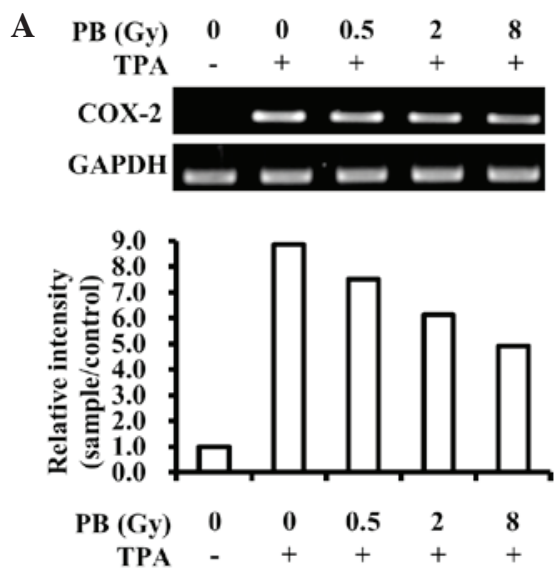
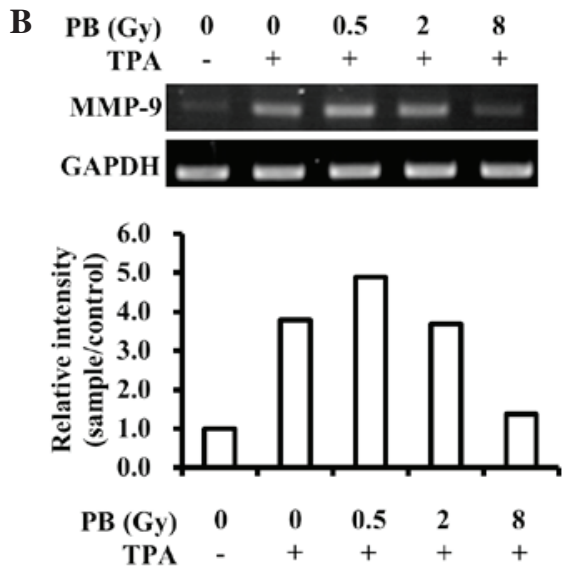

Figure 1. Inhibition of COX-2 and MMP-9 transcription by proton beam irradiation in MDA-MB-213 human breast cancer cells. The cells were irradiated with 0.5, 2 and 8 Gy proton beams prior to TPA treatment for $24 \mathrm{~h}$. (A) COX-2 and (B) MMP-9 expression levels were evaluated by semiquantitative RT-PCR and each band was densitometrically analyzed. The relative intensities of the COX-2 and MMP-9 amplicons are expressed as the fold changes compared with those of the controls and were normalized to GAPDH. COX-2, cyclooxygenase-2; MMP-9, matrix metalloproteinase-9; TPA, 12-O-tetradecanoylphorbol-13-acetate; RT-PCR, reverse transcription polymerase chain reaction; PB, proton beam.
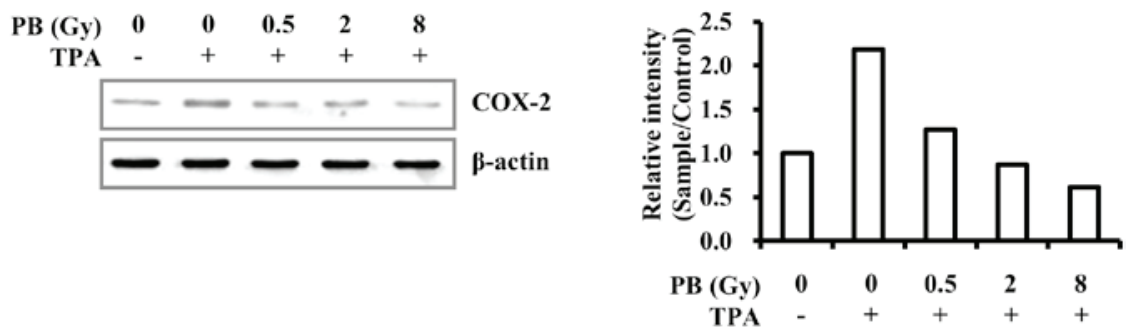

Figure 2. Inhibition of COX-2 protein expression levels by proton beam irradiation in MDA-MB-213 human breast cancer cells. The cells were irradiated with $0.5,2$ and 8 Gy proton beams prior to TPA treatment for $24 \mathrm{~h}$. COX-2 protein expression levels were evaluated by western blotting and each band was densitometrically analyzed. The relative intensity of COX-2 is expressed as the fold change compared with that of the control and was normalized to $\beta$-actin. COX-2, cyclooxygenase-2; TPA, 12-O-tetradecanoylphorbol-13-acetate; PB, proton beam.
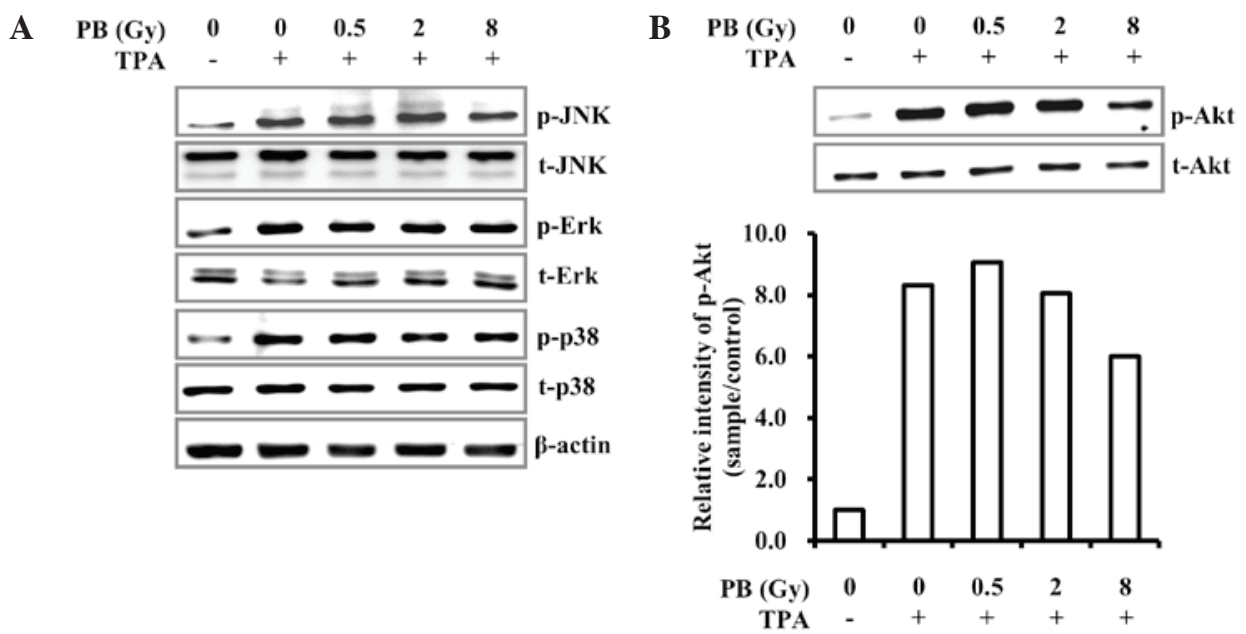

Figure 3. Effects of proton beam irradiation on MAPK and Akt signaling pathways in MDA-MB-213 human breast cancer cells. The cells were irradiated with $0.5,2$ and 8 Gy proton beams prior to TPA treatment for $30 \mathrm{~min}$. The expression levels of (A) p-MAPK, t-MAPK, (B) p-Akt and t-Akt were evaluated by western blot analysis. p-Akt and t-Akt were densitometrically analyzed. The relative intensity of p-Akt is expressed as the fold change compared with that of the control and was normalized to t-Akt. MAPK, mitogen-activated protein kinase; JNK, c-Jun terminal kinase; Erk, extracellular signal-regulated kinase; $\mathrm{PB}$, proton beam.

transcription. Furthermore, COX-2 protein expression levels were dose-dependently suppressed by proton beam irradiation (Fig. 2). These results demonstrate that proton beam irradiation may prevent increases in metastatic potential in MDA-MB-231 triple-negative human breast cancer cells through the downregulation of COX-2 and MMP-9 expression. 


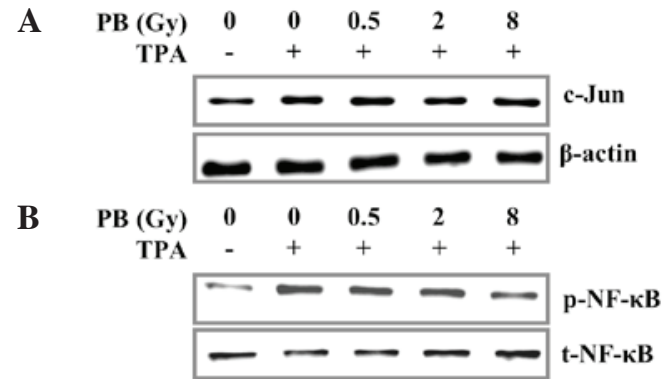

C

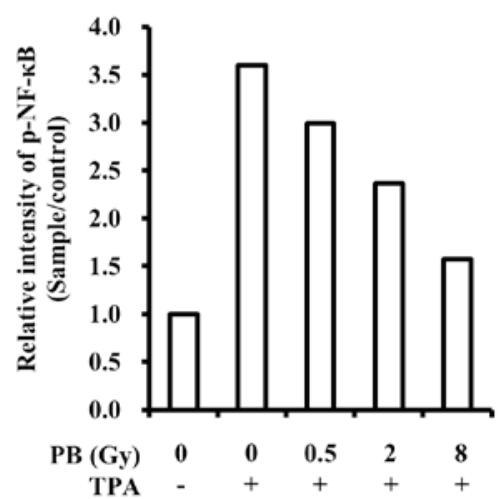

Figure 4. Effects of proton beam irradiation on c-Jun and p-NF- $\kappa \mathrm{B}$ expression levels in MDA-MB-213 human breast cancer cells. The cells were irradiated with $0.5,2$ and 8 Gy proton beams prior to TPA treatment for $30 \mathrm{~min}$. Expression levels of (A) c-Jun, (B) p-NF- $\kappa \mathrm{B}$ and $\mathrm{t}-\mathrm{NF}-\kappa \mathrm{B}$ were evaluated by western blot analysis. $\beta$-actin served as an internal control. (C) p-NF- $\mathrm{B}$ and $\mathrm{t}-\mathrm{NF}-\kappa \mathrm{B}$ were densitometrically analyzed. Relative intensity of $\mathrm{p}-\mathrm{NF}-\kappa \mathrm{B}$ is expressed as fold change compared with that of the control and was normalized to that of $\mathrm{t}-\mathrm{NF}-\kappa \mathrm{B} . \mathrm{p}-\mathrm{NF}-\kappa \mathrm{B}$, phosphorylated nuclear factor- $\kappa \mathrm{B}$; TPA, 12-O-tetradecanoylphorbol-13-acetate; $\mathrm{PB}$, proton beam.
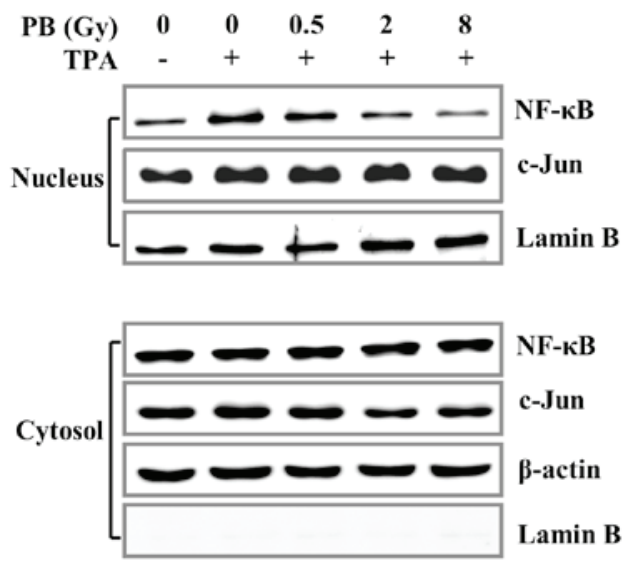

Figure 5. Effects of proton beam irradiation on $\mathrm{c}-\mathrm{Jun}$ and $\mathrm{NF}-\kappa \mathrm{B}$ nuclear translocation in MDA-MB-213 human breast cancer cells. The cells were irradiated with $0.5,2$ and $8 \mathrm{~Gy}$ proton beams prior to TPA treatment for $30 \mathrm{~min}$. c-Jun and NF- $\mathrm{KB}$ levels in the fractions were evaluated by western blot analysis. Lamin $B$ and $\beta$-actin served as internal controls for the nucleus and cytosol, respectively. NF- $\kappa \mathrm{B}$, nuclear factor- $\kappa \mathrm{B}$; TPA, 12-O-tetradecanoylphorbol-13-acetate; $\mathrm{PB}$, proton beam.

Effect of proton beam irradiation on TPA-induced MAPK phosphorylation. TPA-induced expression of COX-2 and MMP-9 is primarily regulated via the PKC/MAPK signaling pathway. Therefore, the effect of proton beam irradiation on the TPA-induced PKC/MAPK signaling pathway was investigated. Despite proton beam irradiation, the phosphorylation levels of the MAPKs, including c-Jun terminal kinase, extracellular signal-regulated kinase and p38, were not changed (Fig. 3A). This result suggests that the reduction in COX-2 and MMP-9 expression levels induced by proton beam irradiation did not involve MAPK signaling.

Effect of proton beam irradiation on TPA-induced Akt phosphorylation. The metastatic activity induced by TPA is also mediated by the PI3K/Akt signaling pathway (15). As shown in Fig. 3A, proton beam irradiation did not prevent TPA-induced MAPK activation. Thus, the change of TPA-induced Akt phosphorylation by proton beam irradiation was investigated. Phosphorylation following TPA stimulation was significantly reduced in MDA-MB-231 human breast cancer cells irradiated by proton beams (Fig. 3B). Akt is a key effector in cancer survival and enhances apoptotic resistance through $\mathrm{NF}-\kappa \mathrm{B}$ activation that induces COX-2, MMP-9 and urokinase-type (u)PA expression $(27,28)$. Consequently, this suggests that proton beam irradiation may prevent COX-2 and MMP-9 expression via downregulation of the Akt signaling pathway.

Effects of proton beam irradiation on c-Jun expression levels, $N F-\kappa B$ phosphorylation and subsequent nuclear translocation. COX-2 and MMP-9 transcription levels are regulated by c-Jun and NF- $\kappa \mathrm{B}$, responsive transcription factors that involve various physiological responses, via binding to cis-acting elements on promoters $(16,29)$. c-Jun and NF- $\kappa \mathrm{B}$ activities are regulated by MAPK and Akt. Akt phosphorylation induced by TPA was effectively reversed by proton beam irradiation in MDA-MB-231 cells (Fig. 3B). Due to this result, the effect of proton beam irradiation on c-Jun expression levels and NF- $\kappa \mathrm{B}$ activation was analyzed. The effects of proton beam irradiation on nuclear translocation of c-Jun and NF- $\kappa \mathrm{B}$ were also investigated. The proton beam irradiation suppressed $\mathrm{NF}-\kappa \mathrm{B}$ activation and subsequent nuclear translocation but not that of c-Jun (Figs. 4 and 5). These results indicate that proton beam irradiation downregulates TPA-induced COX-2 and MMP-9 expression through the inhibition of $\mathrm{NF}-\kappa \mathrm{B}$ activation and subsequent nuclear translocation.

\section{Discussion}

Breast cancer is the primary cause of cancer-related mortality worldwide in females. The five-year survival rate in breast cancer patients depends on whether cancer is localized or metastasized (3). Metastasis is a sign of cancer ingravescence and markedly interferes with cancer therapy (30). Therefore, to treat breast cancer successfully, metastasis requires monitoring. COX-2 activity is closely associated with metastatic potential and tumor growth in cancer. Certain studies have demonstrated that the level of newly synthesized prostaglandin E2 in the blood is associated with tumor growth and metastasis $(11,31)$. Several other studies have observed COX-2 involvement in tumor growth and metastasis, and evidence suggests that COX-2 inhibitors reduced cancer cell growth and metastasis in vitro and in vivo (10-12,31). These studies indicate the importance of COX-2 targeting in cancer therapy. In the present study, proton beam irradiation was found to reduce COX-2 expression levels in MDA-MB-231 invasive human breast cancer cells (Figs. 1A and 2). This suggests that 
proton beam irradiation may prevent cancer progression and metastasis in invasive breast cancer.

During cancer metastasis, degradation of the extracellular matrix (ECM) and basement membrane (BM) is required for the release of cancer cells from the primary tumor and for the attachment of the cells to distant sites. The degradation is catalyzed by membrane proteases, such as MMPs and uPA. MMP-9, one of two gelatinases (MMP-2 and MMP-9), is important in the degradation of ECM and BM in breast cancer metastasis. In addition, poor prognosis and relapse in various cancer patients have been closely associated with MMP-9 overexpression (32,33). MMP-9 not only enhances metastasis but also promotes cancer development and progression. Chang and Werb (34) reported that MMP-9 contributes to cancer proliferation and growth of primary tumors in prostate carcinoma, lymphoma, neuroblastoma and glioblastoma. This suggests that inhibiting MMP-9 expression is important in preventing cancer growth and metastasis. In the present study, proton beam irradiation significantly suppressed the increases in MMP-9 expression levels induced by TPA (Fig. 1B). The result demonstrates that breast cancer growth and metastasis may be inhibited by proton beam irradiation through the inhibition of MMP-9.

COX-2 and MMP-9 expression levels in MDA-MB-231 human breast cancer cells have been shown to be predominantly enhanced by TPA through AP-1 and NF- $\kappa \mathrm{B}$ activation regulated by the PI3K/Akt and/or PKC/MAPK signaling pathways $(32,33)$. Various agents that suppress metastasis and tumor growth downregulate COX-2 and MMP-9 expression via inhibition of the PI3K/Akt and/or PKC/MAPK signaling pathways $(17,19,22)$. The present study demonstrated that proton beam irradiation not only reduced Akt and NF- $\kappa$ B phosphorylation but also inhibited NF- $\kappa \mathrm{B}$ nuclear translocation (Figs. 3B, 4 and 5). However, proton beam irradiation did not affect MAPK phosphorylation or c-Jun transcriptional activity (Figs. 3A, 4 and 5). Therefore, the results suggest that the reduction in TPA-induced COX-2 and MMP-9 expression levels induced by proton beam irradiation is regulated through the inhibition of NF- $\kappa$ B phosphorylation, thus inhibiting subsequent NF- $\mathrm{B}$ nuclear translocation governed by the Akt signaling pathway. In conclusion, the present study indicated that a proton beam may prevent cancer growth and metastasis in triple-negative breast cancer via the suppression of COX-2 and MMP-9 expression through the inhibition of Akt signaling pathway.

\section{Acknowledgements}

This study was supported by the National Research Foundation of Korea grant funded by the Ministry of Science, ICT and Future Planning (grant no. 2012M2B2A4029604).

\section{References}

1. Lim YK, Park BS, Lee SK, Kim KR and Yang TK: A proton beam irradiation method for a uniform dose distribution over a sample volume. J Korean Phys Soc 48: 777-780, 2006.

2. Suit $\mathrm{H}$ and Urie M: Proton beams in radiation therapy. J Natl Cancer Inst 84: 155-164, 1992.

3. Nieves-Alicea R, Colburn NH, Simeone AM and Tari AM: Programmed cell death 4 inhibits breast cancer cell invasion by increasing tissue inhibitor of metalloproteinases-2 expression. Breast Cancer Res Treat 114: 203-209, 2009.
4. Duffy MJ, McGowan PM and Gallagher WM: Cancer invasion and metastasis: changing views. J Pathol 214: 283-293, 2008.

5. Yoon SO, Kim MM and Chung AS: Inhibitory effect of selenite on invasion of HT1080 tumor cells. J Biol Chem 276: 20085-20092, 2001.

6. Chan G, Boyle JO, Yang EK, et al: Cyclooxygenase-2 expression is up-regulated in squamous cell carcinoma of the head and neck. Cancer Res 59: 991-994, 1999.

7. Tucker ON, Dannenberg AJ, Yang EK, et al: Cyclooxygenase-2 expression is up-regulated in human pancreatic cancer. Cancer Res 59: 987-990, 1999.

8. Adhim Z, Matsuoka T, Bito T, et al: In vitro and in vivo inhibitory effect of three Cox-2 inhibitors and epithelial-to-mesenchymal transition in human bladder cancer cell lines. Br J Cancer 105: 393-402, 2011.

9. BoccaC,BozzoF, Bassignana A and Miglietta A: Antiproliferative effects of COX-2 inhibitor celecoxib on human breast cancer cell lines. Mol Cell Biochem 350: 59-70, 2011.

10. Kang JH, Song KH, Jeong KC, et al: Involvement of Cox-2 in the metastatic potential of chemotherapy-resistant breast cancer cells. BMC Cancer 11: 334, 2011.

11. Kundu N and Fulton AM: Selective cyclooxygenase (COX)-1 or COX-2 inhibitors control metastatic disease in a murine model of breast cancer. Cancer Res 62: 2343-2346, 2002.

12. Morita Y, Hata K, Nakanishi M, Nishisho T, Yura Y and Yoneda T: Cyclooxygenase-2 promotes tumor lymphangiogenesis and lymph node metastasis in oral squamous cell carcinoma. Int J Oncol 41: 885-892, 2012.

13. Kim S, Kim SH, Hur SM, et al: Silibinin prevents TPA-induced MMP-9 expression by down-regulation of COX-2 in human breast cancer cells. J Ethnopharmacol 126: 252-257, 2009.

14. Lee KS, Mo, J. Y., Shon, Y. H., Nam, K. S.: Inhibition of metastatic activities in human breast cancer cells irradiated by a proton beam. J Korean Phys Soc 59: 653-656, 2011.

15. Park SY, Kim YH, Kim Y and Lee SJ: Aromatic-turmerone attenuates invasion and expression of MMP-9 and COX-2 through inhibition of NF-kappaB activation in TPA-induced breast cancer cells. J Cell Biochem 113: 3653-3662, 2012.

16. Takahra T, Smart DE, Oakley F and Mann DA: Induction of myofibroblast MMP-9 transcription in three-dimensional collagen I gel cultures: regulation by NF-kappaB, AP-1 and Sp1. Int J Biochem Cell Biol 36: 353-363, 2004.

17. Shin Y, Yoon SH, Choe EY, et al: PMA-induced up-regulation of MMP-9 is regulated by a PKCalpha-NF-kappaB cascade in human lung epithelial cells. Exp Mol Med 39: 97-105, 2007.

18. Lee KS, Shin JS and Nam KS: Starfish polysaccharides downregulate metastatic activity through the MAPK signaling pathway in MCF-7 human breast cancer cells. Mol Biol Rep 40: 5959-5966, 2013

19. Cho HJ, Kang JH, Kwak JY, et al: Ascofuranone suppresses PMA-mediated matrix metalloproteinase- 9 gene activation through the Ras/Raf/MEK/ERK- and Ap1-dependent mechanisms. Carcinogenesis 28: 1104-1110, 2007.

20. Garg A and Aggarwal BB: Nuclear transcription factor-kappaB as a target for cancer drug development. Leukemia 16: 1053-1068, 2002.

21. Pastore S, Mascia F, Mariotti F, Dattilo C, Mariani V and Girolomoni G: ERK1/2 regulates epidermal chemokine expression and skin inflammation. J Immunol 174: 5047-5056, 2005.

22. Park SK, Hwang YS, Park KK, Park HJ, Seo JY and Chung WY: Kalopanaxsaponin A inhibits PMA-induced invasion by reducing matrix metalloproteinase-9 via PI3K/Akt- and PKCdelta-mediated signaling in MCF-7 human breast cancer cells. Carcinogenesis 30: 1225-1233, 2009.

23. Cragg GM, Newman DJ and Weiss RB: Coral reefs, forests, and thermal vents: the worldwide exploration of nature for novel antitumor agents. Semin Oncol 24: 156-163, 1997.

24. Jang JY, Jeon YK and Kim CW: Degradation of HER2/neu by ANT2 shRNA suppresses migration and invasiveness of breast cancer cells. BMC Cancer 10: 391, 2010.

25. Degner SC, Kemp MQ, Bowden GT and Romagnolo DF: Conjugated linoleic acid attenuates cyclooxygenase-2 transcriptional activity via an anti-AP-1 mechanism in MCF-7 breast cancer cells. J Nutr 136: 421-427, 2006.

26. Guttilla IK, Phoenix KN, Hong X, Tirnauer JS, Claffey KP and White BA: Prolonged mammosphere culture of MCF-7 cells induces an EMT and repression of the estrogen receptor by microRNAs. Breast Cancer Res Treat 132: 75-85, 2012.

27. Sheng S, Qiao M and Pardee AB: Metastasis and AKT activation. J Cell Physiol 218: 451-454, 2009. 
28. Agarwal A, Das K, Lerner N, et al: The AKT/I kappa B kinase pathway promotes angiogenic/metastatic gene expression in colorectal cancer by activating nuclear factor-kappa B and beta-catenin. Oncogene 24: 1021-1031, 2005.

29. Kumar R, Alam S, Chaudhari BP, et al: Ochratoxin A-induced cell proliferation and tumor promotion in mouse skin by activating the expression of cyclin-D1 and cyclooxygenase-2 through nuclear factor-kappa B and activator protein-1. Carcinogenesis 34 647-657, 2013.

30. Shao W, Wang D and He J: The role of gene expression profiling in early-stage non-small cell lung cancer. J Thorac Dis 2: 89-99, 2010.
31. Altundag K and Ibrahim NK: Aromatase inhibitors in breast cancer: an overview. Oncologist 11: 553-562, 2006.

32. Kupferman ME, Fini ME, Muller WJ, Weber R, Cheng Y and Muschel RJ: Matrix metalloproteinase 9 promoter activity is induced coincident with invasion during tumor progression. Am J Pathol 157: 1777-1783, 2000.

33. Stamenkovic I: Matrix metalloproteinases in tumor invasion and metastasis. Semin Cancer Biol 10: 415-433, 2000.

34. Chang $\mathrm{C}$ and Werb Z: The many faces of metalloproteases: cell growth, invasion, angiogenesis and metastasis. Trends Cell Biol 11: S37-S43, 2001. 